quarischen Gesellschaft Graubündens XLIX, 1919. 50 SS. in $8^{\circ}$.

Dr. Fritz Jecklin, Die Churer Waisenpflege bis in die Neuzeit. Festgabe zur Feier des 75 jährigen Bestehens der bürgerlichen Waisenanstalt in Chur. Chur, Graphische Anstalt Manatschal, Ebner \& Cie. 1920. 110 SS. in kl. $8^{n}$ mit einem Titelbild.

Hans Roth, Die religiöse Kinder-Erziehung nach schweizerischem Recht. Zürcher jur. Diss. 1920. Zürich, Gebr. Leemann \& Cie. o. J. $99 \mathrm{SS}$. in $8^{0}$.

Dr. jur. Anna Margaretha Schlatter, Der Schutz der ehelichen Gemeinschaft. Zürcher jur. Diss. 1920. BornaLeipzig, Robert Noske 1920. VI und $114 \mathrm{SS}$. in $8^{\circ}$.

Dr. jur. Robert von Stürler, Fürsprecher in Bern, Die vier Berner Landgerichte Seftigen, Sternenberg, Konolfingen und Zollikofen. Berner jur. Diss. 1920. Borna-Leipzig, Robert Noske, Bern, A. Francke 1920. XIV und $287 \mathrm{SS}$. in $8^{\prime \prime}$. Georg Felix Bein, Die historische Entwicklung der Leinwandweberei im Kanton Bern mit besonderer Berücksichtigung der ländlichen Meisterschaftsverbände. Berner staatswiss. Diss. 1920. o. O. u. J. VIII und $108 \mathrm{SS}$. in $8^{\circ}$ mit zwei Bildern.

Festgabe zur LXXIII. Jahresversammlung (Langenthal, 7.;8. September 1919) der Allgemeinen geschichtforschenden Gesellschaft der Schweiz, dargeboten vom Historischen Verein des Kantons Bern. Bern, Buchdruckerei Dr. Gustav Grunau 1920, 262 SS. in $8^{\circ}$.

Dr. Eduard His, Privatdozent der Rechte an der Universität Basel, Geschichte des neuern Schweizerischen Staatsrechts. Erster Band: Die Zeit der Helvetik und der Vermittlungsakte 1798-1813. - Basel, Helbing \& Lichtenhahn 1920. XX und 692 SS. in $8^{\circ}$.

Andreas Heusler, Schweizerische Verfassungsgeschichte. Basel, Frobenius A.-G. 1920. VIII und 392 SS. in $8^{\circ}$.

Ulrich Stutz, Die Schweiz in der Deutschen Rechtsgeschichte. Wissenschaftliche Festrede, gehalten in der öffentlichen Sitzung der Preußischen Akademie der Wissenschaften in Berlin zur Feier des Jahrestages König Friedrichs II. 
Sonderdruck aus den Sitzungsberichten 1920 IV S.92-114, vermehrt um ein Verzeichnis von Schriften des Verfassers zur Schweizerischen Rechtsgeschichte. Berlin, in Kommission bei der Vereinigung wissenschaftlicher Verleger Walter de Gruyter \& Co. 1920. 23 SS. in gr. $8^{\circ}$.

Bei den Schwierigkeiten, auf die infolge des üblen Standes der deutschen Valuta der Bezug ausländischer Literatur heutzutage stößt, wäre eine ausführliche Berichterstattung über solche erwünschter als je. Allein die Beschränkung, die wir uns wegen der Teuerkeit von Satz und Papier auferlegen müssen, zwingt uns leider umgekehrt, in unserem diesjährigen Schweizer-Bericht über die zahlreich uns zugegangenen Veröffentlichungen im einzelnen uns noch kürzer als sonst zu fassen, obwohl das vergangene Jahr für die Schweizerische Rechts- und Verfassungsgeschichte besonders ertragreich war und Werke gezeitigt bat, die auf lange hinaus grundlegend bleiben dürften.

Wir beginnen mit einigen kleineren Studien zur Stadt- und Stadtverfassungsgeschichte. Seine zahlreichen Beiträge zur Baugeschichte und überhaupt zur Geschichte Berns vermehrte Eduard von Rodt um einen neuen, der in einem ersten Teil die Nydegg behandelt, jene, während des Interregnums zerstörte, in den usurpierten Freiheiten der gefälschten Handfeste sowie in einem Gnadenerlaß Rudolfs von Habsburg von 1274 erwähnte, von Justingers Berner Chronik als schon vor der Stadtgründung vorhanden angenommene Burg auf der niedersten Ecke der Aarehalbinsel, auf der nachmals Bern erbaut wurde. Der zweite Teil befaßt sich, wiederum im Anschluß an Justinger und durch andere Nachrichten sowie spätere Befunde ihn zu erhärten suchend, mit der Gründungsgeschichte der Stadt (ein Bubenberg als Iocator? Stadtanlage, Hofstätten, Name, Stadtsiegel, Pfarrzugehörigkeit). Alles in allem ein sehr lehrreicher Beitrag zur Geschichte der deutschen Städtegründung.

In der ersten seiner woblabgewogenen, auch für die Deutsche Rechtsgeschichte überhaupt belangreichen Untersuchungen beschäftigt sich Jacob Wackernagel mit einer zunächst sehr auffälligen, wenn auch durchaus nicht ganz vereinzelt dastehenden Maßregel Rudolfs von Habsburg. Gleich nach seiner Wahl zum deutschen König entzog er nämlich dem Bischof von Basel die diesem seit etwa einem Jahrhundert zustehende Stadtvogtei, um sie als Reichsvogtei seinem habsburgischen Dienstmann und Vertrauten Hartmann von Baldegg zu übertragen, wodurch Basel in die Gefahr kam, eine österreichische Landstadt zu werden. Wackernagel weist nun überzeugend nach, daß der Grund für dies Vorgehen das Bestreben war, nicht nur zwischen den linksrheinischen Besitzungen des habsburgischen Hauses in der heutigen Schweiz sowie im Elsaß und den rechtsrheinischen Herrschaften, die ihm gehörten, eine Brücke zu schlagen und sich das besonders wichtige Mittelstück zu sichern. Vielmehr bedurfte Rudolf Basels namentlich für die uechtländische Territorialpolitik gegenüber Savoyen, um ein 
störendes Eingreifen in diese von Deutschland her zu hindern. In der zweiten Studie verfolgt Wackernagel für Basel und Zürich unter Berücksichtigung von Luzern und Straßburg den Aufstieg des Rats von einer bloßen Gemeindevertretung zu einer auch staatliche Aufgaben wahrnehmenden Behôrde. Für Basel scheint mir die Stufenleiter: ausschließlich Gemeinderat, daneben von Gemeinde wegen gestelltes Urteilsfinderkolleg für die stadtherrlichen Gerichte, weiter Inhaber gewisser Befugnisse vogteilicher Gerichtsbarkeit auch ohne Vogtsvorsitz, schließlich durch das Medium des Stadtfriedens ausschließliche Jurisdiktion für bestimmte Straftaten, in der Hauptsache einwandfrei nachgewiesen zu sein. Für Zürich dürfte dagegen der Richtebrief doch eher die bisherige Ansicht stützen, wonach die vom Rat ausgesprochenen Strafen zu den etwa im Vogteigericht schon verhängten hinzukamen; namentlich für Rb. I 4 und I 16 vermag ich mich der von dem Verfasser vorgeschlagenen neuen Deutung nicht anzuschließen.

Gleich in diesem Zusammenhang sei auf das letzte Basler Neujahrsblatt hingewiesen, in dem August Burckhardt an Hand eines reichen Materials die Zusammensetzung der Basler Bürgerschaft von den ältesten Zeiten bis zum Ende des 18. Jahrhunderts schildert. Die ersten zwei Drittel der Arbeit, die das Mittelalter behandeln, machen uns mit Zeiten bekannt, in denen die Zuwanderung verhältnismäßig nicht geringer war als in den letzten Jahrzehnten, undlehren uns Basel als das am meisten demokratische städtische Gemeinwesen der heute schweizerischen Gebiete kennen. Zur Aufrichtung einer Geburts- oder einer Geldaristokratie gelangte man in Basel auch später nicht, wohl aber zu einem Syndikat von Großkaufleuten und Fabrikanten nebst einigen aus fremden Kriegsdiensten zurückgekehrten Militärs und etlichen Juristen, das tatsächlich das Regiment in den Händen hatte. Gerade die Angaben, die Burckhardt über diese in die höchst demokratische Form eines reinen Zunftregiments sich kleidende, fast ausschließliche Herrschaft von etwa zwei Dutzend Familien, über die Revolution von 1691 und über die Ratsund Ämterbesetzung seit ihr macht, interessieren von dem schönen Gesamtbilde, das er zeichnet, mit am meisten.

Karl Meyers tief schürfende Studien zur Rechts-, Wirtschaftsund Sozialgeschichte der südlichen Alpentäler, die er in seinen Büchern über Blenio und Leventina ${ }^{1}$ ) und über die Capitanei von Locarno ${ }^{2}$ ) niedergelegt hat, zeitigten neuestens als mittelbares Ergebnis auch einige dankenswerte Beiträge zur Geschichte der ersten Schweizer Bünde. ${ }^{3}$ ) Unter diesen ragt ein 1917 vor der Schweizerischen allgemeinen geschichtforschenden Gesellschaft gehaltener und jetzt im Jahrbuch für Schweize-

1) Vgl. diese Zeitschr. XXX, 1912, Germ. Abt. S. $653 \mathrm{ff}$.

2) S. diese Zeitschr. XXXVIII, 1917, Germ. Abt. S. 446 ff.

3) Vgl. diese Zeitschr. XXXVII, 1916, Germ. Abt. S. 720 sowie die Aufsätze,,Der Schwurverband als Grundlage der schweizerischen Eidgenossenschaft" im Anzeiger f. Schweizer. Geschichte, 1919, S. 183 ff. und „Die Einwirkung des Gotthardpasses auf die Anfänge der Eidgenossenschaft:", Geschichtsfreund LXXIV, 1919, S. $257 \mathrm{ff}$.

Zeitschrift für Rechtsgeschichte. XLI. Germ. Abt. 
rische Geschichte gedruckt vorliegender Vortrag hervor, der die Einwirkung italienischer Einflüsse bei der Entstehung der Eidgenossenschaft sehr wahrscheinlich macht. Von einem bündigen Beweis kann natürlich nicht die Rede sein, da es an Quellen für den Nachweis des Zusammenhangs bisher fehlt, und da auch die rechts- und wirtschaftsgeschichtlichen Tatbestände nicht so sind, daß die daraus gezogenen Schlüsse zwingend wären. Aber der Gedanke hat etwas außerordentlich Bestechendes, und es ist ein unbestreitbares Verdienst Meyers, zum erstenmal darauf hingewiesen zu haben, daß näher als die Annahme einer Einwirkung der deutschen Städtefreiheit die einer solchen von seiten der oberitalienischen, auch die Landschaft ergreifenden Kommunalbewegung liegt. Meyers Studie, die außerdem im einzelnen manche beachtenswerte Beobachtung und Anregung enthält, wird sicherlich die gebührende Beachtung finden und dazu beitragen, die so viel erörterten Anfänge der schweizerischen Eidgenossenschaft wieder in neuem Lichte erscheinen zu lassen.

Schon 1918 erschien, aber jetzt erst ging uns zu die Studie von Rosa Benz über die Verfassung des Landes Appenzell bis 1513. Man wird kaum irregehen, wenn man sie als eine Ergänzung der früheren Arbeit der Verfasserin über das Landammannamt in der Urschweiz ${ }^{1}$ ) ansieht, die, da die Geschichte dieses Amtes und der Landsgemeinde in diesem Falle für eine selbständige Untersuchung nicht ausreichte und auch für sich allein kaum sich behandeln ließ, weitergreifend überhaupt die ältere Verfassungsentwicklung Appenzells ins Auge faßte. Aus den die Grundherrschaft des Klosters St. Gallen und die schließlich an dessen Abt übergehende hohe Vogtei behandelnden ersten beiden Kapiteln hebe ich nur hervor die schon im 15. Jahrhundert als Steuerbezirke bezeugten 6 Roden mit ihren Vorstehern, den Rodmeistern und deren Gehilfen, den Samnern, sowie die Klasse der homines speciales ecclesie sci. Galli vulgariter dicti Sunderlüte (1288), die mit ihren zerstreut liegenden, namentlich im Amt Gossau, in Gais, in Herisau, Teufen und Speicher begegnenden Sondergütern ein Sonderamt bilden, unter keiner Vogtei, vielmehr direkt unter dem Abt stehen, von diesem ihren Ammann für ihr Gericht erhalten und jenem ehrschatzptlichtig sind, also vielleicht Insassen der engeren Immunität. Von einer Talschaft Appenzell ist zuerst 1333 die Rede, 1346 auch von den Landleuten von Appenzell, die dann bald einheimische Ammänner an Stelle von dienstmännischen des Abtes erhalten. Die Anlehnung an den schwäbischen Städtebund bringt die Wahl von 13 Vorstehern und damit den ersten Zusammentritt einer Landsgemeinde mit sich (1378). Die ersten Landammänner dagegen wurden den Appenzellern gleich dem Kriegshauptmann seit 1404 von den mit ihnen gegen den Abt von St. Gallen verbündeten Schwyzern gestellt. Erst 1417 ist in der Person von Ulrich Entz ein einheimischer Landammann bezeugt. Dieser wird aus gewissen angesehenen Geschlechtern jeweilen auf ein Jahr gewählt, behält aber auf Grund immer wieder vorgenommener Neuwahl sein Amt mitunter längere Zeit, ge-

1) Vgl. diese Zeitschr. XL, 1919, Germ. Abt. S. 378f. 
legentlich Jahrzehnte hindurch. Im übrigen ist für die Landesverfassung offensichtlich das Muster von Schwyz maßgebend gewesen. Der ebenso fleißigen wie zuverlässigen Arbeit, deren Hauptwert in einer übersichtlichen Zusammenarbeitung des appenzellischen Materials besteht, sind Ammännerlisten beigegeben.

Die stattliche Zahl der die Geschichte der einzelnen Markgenossenschaften der Schweiz und ihrer Ausläufer behandelnden Untersuchungen wird von Ernst Meyer um eine neue vermehrt, welche die Nutzungskorporationen im sog. Freiamt, seit 1415 Untertanenland von sechs (später sieben) eidgenössischen Orten, seit 1803 ein Teil des Kantons Aargau, behandelt. Sie holt nach der Art solcher Erstlingsarbeiten etwas weit aus. Doch ist dabei zu berücksichtigen, daß der Verfasser nicht bloß an die Fachgelehrten sich wendet, sondern auch an weitere historisch und juristisch interessierte Kreise, und gerne sei anerkannt, daß seine Abhandlung, der sich Fritz Fleiner besonders angenommen hat, mit Geschick und Geschmack ihre Aufgabe bewältigt. Sie bedeutet schon um deswillen eine Bereicherung unserer Literatur, als sie besonders gut bezeugte Markgenossenschaften aus dem schweizerischen Flachland zum Gegenstand hat, und zwar des näheren zwei große (Hundertschaftsmarken) und mehrere kleine, über deren ursprüngliches Verhältnis zueinander freilich nicht mehr ins klare zu kommen ist. Die großen Marken lösten sich allerdings verhältnismäßig früh auf, nur die gemeinsame Weidenutzung für die in ihrem ehemaligen Bereich liegenden Ortschaften erinnerte später noch daran. Neben unfreien, neben HofGemeinden begegnen namentlich auch solche Freier - der Name Freiamt bürgert sich freilich erst seit 1500 ein - und gemischte. Was die Einzelheiten anlangt, so ergeben sie naturgemäß nicht sowohl neue Züge, als vielmehr hie und da erwünschte genauere Aufschlüsse oder lehrreiche Belege. Beachtung verdienen z. B. die Feststellungen S. $60 \mathrm{f}$. über das Verhältnis der Einzelhöfe zu den Dorfverbänden, S. $89 \mathrm{ff}$. über die Stadt Bremgarten und ihre Allmende, S. 114 über das Dorfrecht nach auswärts Verzogener und seine Vererblichkeit, S. $118 \mathrm{ff}$. über die Umbildung zur Realgemeinde und die „Gerechtigkeiten"1 ${ }^{1}$, die aber regelmäßig mit den Häusern verbunden blieben, bis die Helvetik den Schritt zur Rechtsamegemeinde tat, S. $125 \mathrm{ff}$. über Hubgemeinden, S. $131 \mathrm{ff}$. über das Amt Merenschwand, dessen Leute sich 1393/4 von der Eigenschaft und dem Zwing und Bann der Edlen von Hünenberg loskauften und als Ausbürger Luzerns mit eigenem Amtsreeht bis zur Neuzeit eine reine Personalnutzungsgemeinde bildeten, S. 145 über Aufhebung genossenschaftlicher Weiderechte und Allmendaufteilungen im 18. Jahrhundert. Zum Schluß wird die Entwicklung seit der Helvetik geschildert und das geltende Recht dargestellt. Danach sind heute die aargauischen Gerechtigkeiten wohlerworbene Privatręchte auf Nutzung von Pflanzplätzen und Wald und mit der Verpflichtung zu Hand- und Spanndiensten und

1) Vgl, neuestens über ,Rechtsame und Gerechtigkeiten" Mas G mü r in der Festgabe für Philipp Lotmar, Bern 1920 (auch sep.). 
unter Umständen zu Geldleistungen. Die Gerechtigkeitsgemeinde, nunmehr überall eine Rechtsamengemeinde, hat ihr früheres Eigentum an die Bürgergemeinde verloren und ist auf ein dingliches Recht an der Allmende beschränkt. Die Bürgergemeinde aber, eine Personalgemeinde und wohl zu unterscheiden von der Einwohnergemeinde, der politischen und administrativen Gemeinde, ist außer für das Bürgerrecht und die Armenverwaltung nur noch da für die Verwaltung und die Nutzung ihrer Bürgergüter (Allmende), welch letztere in der Gestalt des jederzeit durch die Gemeindeversammlung abänderlichen, ja aufhebbaren „Bürgernutzens" erfolgt. Dies in Kürze über die Schrift Meyers, die einen neuen Beleg für die in der Schweiz oft erprobte Nützlichkeit der Beschäftigung von Doktoranden mit der Rechtsgeschichte ibrer engsten Heimat liefert.

In diesem Zusammenhang mag einer in Freiburg i. Ue. unter der Leitung von Richard Zehntbauer entstandenen Arbeit über die ehemaligen stadtzugerischen Fischereirechte gedacht werden, die einen jetzt als Rechtsanwalt tätigen Zuger zum Verfasser hat. Gleich der vorgenannten von Meyer stützt sie sich außer auf gedrucktes Material auch auf archivalisches. Der historische Teil zerfällt in drei Kapitel. Ihr erstes behandelt den ,,unverlehnten“ See, das der Stadt vorgelagerte Seestück, in dem es seit dem Loskauf der Fischzehnten durch die Stadt zu Ende des 14. Jahrhunderts keine einzelnen Fischenzen mehr gab. Vielmehr wurde er von den bereits in jener Zeit zu einer Fischerbruderschaft zusammengeschlossenen Zuger Fischern beworben. Jetzt gehört die Fischerei in ihm dem Kanton, nachdem vor einem Vierteljahrhundert ein im Streit zwisehen Stadt und Staat ergangenes bundesgerichtliches Urteil die durch Helvetik und Mediation arg verwirrte Rechtslage in diesem Sinne klären zu müssen geglaubt hat. Ich übergehe die einzige auf dem Ostufer daran anstoßende „Eiola“-Fischenz, ehedem mit dem gegenüberliegenden Böschenroth im Obereigentum des Stifts Beromünster, das dort Zwing und Bann und die Niedergerichtsbarkeit hatte. Am westlichen Ufer liegen die von der Stadt gegen einen Pachtschilling verliehenen, Lehensseen genannten Fischenzen grundherrlichen Ursprungs, die einen ehedem zu dem 858 von Ludwig dem Deutschen an das Fraumünsterstift in Zürich geschenkten Königshof Cham gehörig, der 1477 von der Stadt Zug gekauft wurde, andere einst dem Kloster Muri durch seine Höfe in Gangolfswil und Dersbach zuständig, nachmals aber alle von Zug erworben. Auf das einzelne kann nicht eingegangen werden. Bemerkt sei nur, daß alle diese Fischereirechte in neuerer Zeit zu Rechtsstreitigkeiten Anlaß gegeben haben, über die Iten eingehend berichtet. Den Schluß des historischen Teils macht ein Kapitel über das altzugerische Fischereirecht, das, soweit es sich um den unverlehnten See handelte, als ein Teil der Allmendnutzung behandelt wurde, sowie ein Anhang mit bisher ungedruckten Fischerordnungen. Bezüglich des dogmatischen Teils, in dem der Verfasser die Zuger Fischenzen als beschränkte dingliche Rechte am öffentlichen Gewässer erklärt, die veräußerlich und vererblich seien und ein ausschließliches Aneignungsrecht gewähren, muß 
ich mich auf einen bloßen Hinweis beschränken. Das Ganze ist eine recht brauchbare und dankenswerte Untersuchung.

Aus mehreren die kirchlichen Dinge betreffenden Abhandlungen, die mir zugegangen sind, hebe ich heravs die Schrift von Elsa Beurle über den politischen Kampf um die religiöse Einheit der Eidgenossenschaft 1520-27. Die Reformation hatte die religiöse Einheit der Schweiz, die schon vorher namentlich durch die Zürcherische Kirchenpolitik ins Wanken gekommen war, zunächst aufgehoben. Die katholischen Orte wollten sie von 1520 an nicht bloß für die gemeinen Herrschaften, sondern im ganzen mit Hilfe der Tagsatzung, auf der sie die Mehrheit hatten, im Sinne des alten Glaubens wiederherstellen, Zwingli sie im Sinne des neuen aufrichten, 1520-1523 mehr nur mit geistigen Mitteln, zuletzt 1528 bis zu seinem Tode mit Gewalt, dazwischen 1524-1527 durch Verwirklichung eines staatsmännischen Programms, das die Schweizer Bünde und infolgedessen auch die Tagsatzung in Glaubensangelegenheiten für neutral erklärte. Diesem Kampf um die Frage, ob die Tagsatzung über Religionsstreitigkeiten entscheiden dürfe oder nicht, ist in der Hauptsache die von Walther Köhler angeregte Schrift unserer Verfasserin gewidmet. Zur Rechtsgeschichte selbst trägt sie nichts bei, da ja beide Teile nicht zum Ziele gelangten. Aber die politische Seite dieses Ringens gelangt gut zur Darstellung. Mittelbar kommt die Arbeit immerhin auch für die Verfassungsgeschichte der Schweiz in Betracht.

$\mathrm{Zu}$ dem im Jahre 1436 abgeschlossenen Zehngerichtenbund gehörte anfänglich als elftes Bundesglied das Chorherrengericht zu Schiers im Prätigau, entstanden auf Grund einer Schenkung Graf Ottos von Kirchberg, die er 1209 an das Domkapitel von Chur gemacht hatte. Es ist aber beileibe nicht 'etwa ein Beitrag zur Geschichte der kirchlichen Gerichtsbarkeit, vielmehr ein solcher zur Gesehichte des Churer Kirchenguts, den Prof. Dr. Constanz Jecklin in der darauf bezüglichen Schrift bietet, worin er aus dem Besitz der Historisch-Antiquarischen Gesellschaft Graubündens Regesten und Urkunden dieses Kapitels- oder Herrengerichts publiziert und mit einer Einleitung versieht. Letztere verbreitet sich außer über das Kapitelsgut in Schiers und im übrigen Prätigau sowie über das Amt des Kapitelsammanns namentlich auch über die Kapitelsleute und zeigt, wie ihr Gericht, das ein Niedergericht war, auch in privatrechtlichen Dingen mehr und mehr dem Herrschaftsgericht Schiers und Seewis weichen mußte, dem ursprünglich nur die Untertanen der Grafen von Toggenburg, später der Matsch, schließlich Österreichs zugehörten. M. a. W. die Kapitelsleute gingen in den Herrschaftsleuten auf. Immerhin erhielt sich noch bis ins 17. Jahrhundert für jene ein eigenes Zivilgericht. Doch wird 1680 bei der Teilung des Hochgerichts Schiers in die beiden Gerichte Schiers und Seewis einer Sonderstellung der Kapitler nicht mehr gedacht.

Ein Gegenstand, der es verdiente, einmal in großem Stil rechtsgeschichtlich bearbeitet zu werden, sind die Fremdenherbergen, Hospitäler, Siechenhäuser, Waisenhäuser usw. An wertvollen, auch juristisch ertragreichen Untersuchungen über einzelne von ihnen fehlt es nicht. 
Vor allem dem 18. und dem 19. Jahrhundert ist freilich gewidmet die Schrift des Churer Staatsarchivars Dr. Fritz Jecklin über die Churer Waisenpflege. ${ }^{1}$ ) Ganz kurz werden in ihr die Churer Spitäler des Mittelalters behandelt, ausführlich mehrere erfolglose, z. T. an dem Widerstand der Zünfte gescheiterte Versuche des 18. Jahrhunderts, in Chur ein Zucht- und Waisenhaus zu errichtèn und die dafür erforderliche Organisation zu schaffen. Schließlich führte aber doch die Notwendigkeit, dem zur Landplage gewordenen Bettler- und Landstreichertum zu steuern, 1786 zur Gründung einer städtischen Armenanstalt in Chur, in die gelegentlich auch Waisenkinder, die man im übrigen bei Bauersleuten zu verdingen vorzog, Aufnahme fanden. Eine besondere Waisenanstalt wurde im Zusammenhang mit der Aufteilung und Urbarmachung der Allmende 1841 in Ober-Masans eingerichtet. Die Schrift ist zugleich als Beitrag zur Geschichte des städtischen Pflegschaftswesens und der Beteiligung der Zünfte an der Stadtverwaltung lehrreich.

In der alten Eidgenossenschaft herrschte nach der Glaubenstrennung innerhalb der einzelnen Orte Glaubenszwang mit Ausnahme von einigen wenigen aus ihnen sowie von gewissen von Katholischen und Reformierten gemeinsam regierten Vogteien, für die der Grundsatz der Parität sich durchsetzte. Ein paritätisches regierendes Ort war Glarus; nach seinem Recht sollten Kinder aus gemischten Ehen samt und sonders in der Religion des Vaters auferzogen werden. Die Teilung der Kinder nach dem Geschlecht kannte Graubünden und vielleicht auch Appenzell vor der Trennung von 1597 in die beiden konfessionell geschlossenen Inner. und Außerroden, namentlich aber das santgallische Untertanenland Toggenburg seit 1723; sie war seit der zweiten Hälfte des 18. Jahrhunderts Rechtens auch für die gemeinen Vogteien. Helvetik und Mediation brachten eine Vermehrung der Zahl der paritätischen Stände und eine Abschwächung der konfessionellen Geschlossenheit anderer. Eine gemeineidgenössische Regelung der konfessionellen Kindererziehung auf dem Wege einer Einigung unter den souveränen Kantonen gelang nicht. In den Kantonen ging man von dem Grundsatz der Trennung der Kinder nach dem Geschlecht ab und ließ entweder die in dem betreffenden oder im Heimatkanton herrschende Staatsreligion oder die Konfession des Vaters maßgebend sein oder das freie Ermessen des Vaters, eine Lösung, die unter der Herrschaft der Bundesverfassung von 1848 durch das Bundesgesetz vom 3. Dezember 1850 eidgenössisches Recht wurde und schließlich auch in Art. 49 Abs. 3 der Bundesverfassung von 1874 überging. Das Nähere ergibt sich aus der zu einem wesentlichen Teil auf Fleiners Untersuchungen über die Parität in der Schweiz fußenden, sie aber ergänzenden Abhandlung von Roth, auf deren historischen Abschnitt unsere Leser hiermit verwiesen seien.

Ebenfalls auf das geltende Recht, also auf die Behandlung des Schutzes der ehelichen Gemeinschaft im Schweizerischen Zivilgesetz-

1) Von demselben vgl. auch Mitteilungen über die Frauenkloster. kirche St. Peter zu Müstail bei Alvaschein, Chur, A. G. Bündner Tagblatt 1920,8 SS. in $8^{\circ}$ mit Titelbild. 
buch zielt ab die fleißige Arbeit von Margaretha Schlatter. Doch auch sie unterläßt es nicht, in einem ersten Teil die geschichtliche Entwickelung dem Leser vorzuführen, und zwar vom alamannischen Volksrecht an bis herab auf die kantonalen Gesetzbücher des vorigen Jahrhunderts. Schon früher wurde eben der Versuch gemacht, die eheliche Gemeinschaft durch richterliches Eingreifen zu schützen. Besonders verdienstlich ist, was die Verfasserin auf S. 24-42 über das zürcherische Recht des Reformationsjahrhunderts und der Folgezeit ausführt. Sie stützt sich daher auf Eglis Aktensammlung zur Zürcher Reformationsgeschichte und die dort und anderwärts gedruckten Thesatzungen von $1525,1698,1719$, vor allem aber auch auf ungedrucktes Material im Zürcher Staatsarchiv, insbesondere auf die Protokolle und Weisungen des Ehegerichts und auf die Ratsmandate. Was sie daraus über die Organisation, über das Verfahren und die Praxis des zürcherischen Fhegerichts, über die Einrichtung der ,,Fhegaumer" und ,, Stillstände ", über die in Zürich besonders ausgebildete zeitliche Trennung von Tisob und Bett, über den völligen Verzicht auf direkten und über die Anordnung indirekten $\mathbf{Z}_{\text {wangs }}$ beibringt, verdient von jedem beachtet zu werden, der sich mit der Geschichte des evangelischen und staatlichen Eherechtes befaßt, stellt sich aber vor allem auch dar als ein recht nützlicher und dankenswerter Beitrag zur Geschichte des schweizerischen öffentlichen und privaten Rechts.

Mehrere tüchtige Beiträge zur Rechts- und Verfassungsgeschichte des Berner Herrschaftsgebiets sind aus den letzten Monaten zu verzeichnen. Ich beginne mit Robert von Stürlers Untersuchung. Die Landgrafschaft Burgund zerfiel schon früh in zwei Teile. Links der Aare lag, hervorgegangen aus der Grafschaft Bargen, die Landgrafschaft AarBurgund, durchsetzt mit ziemlich viel Reichsland und bei der Eroberung durch Bern 1388 in die Landgerichte Seftigen und Sternenberg zerfallend. Die Landgrafschaft Klein-Burgund auf dem rechten Aareufer ging 1406 an Bern über; sie umfaßte vier Landgerichte, darunter die von Konolfingen und Zollikofen. Die vier genannten Landgerichte der beiden Landgrafschaften schlossen als innere Vogteien der Stadt, um die sie in weitem Umkreise herumlagen, eine Menge kleiner geistlicher und weltlicher Twingherrschaften und mehr als vierzig Kirchspiele in sich ein. Über sie waren die vier Venner der Stadt und anfänglich auch Freiweibel als Unterbeamte gesetzt; darunter standen Ammänner. Stürler gibt zunächst eine genaue Beschreibung der vier Landgerichte, ihrer Gerichtsstätten, ihres Gerichtsbezirks, ihrer Unterbezirke und Herrschaften, und macht uns mit ihren ursprünglichen Inhabern bekannt. Die in ihnen begegnenden Herrschaften standen teils geistlichen, teils weltlichen Herren zu, von denen den einen sogar die Blutgerichtsbarkeit zukam, den andern nur die niedere Gerichtsbarkeit, der Twing und Bann. Ihre Herren hießen danach Twingherren, deren Herrschaften Twingherrschaften. Darüber erhob sich die von Bern erworbene landgräfliche Gewalt, insbesondere Gerichtsbarkeit. Jedoch sie reichte, da Bern deren Rechte übernahm, wie es sie vorgefunden hatte, in den einzelnen Fällen 
verschieden weit. Natürlich suchte Bern sie zu konsolidieren und, zumal nach dem Freiheitsbrief, den ihm König Sigismund 1415 erteilt hatte, die landesherrliche Gewalt stärker zu betonen. Jedoch das stieß auf Schwierigkeiten. Nach Auffassung der Herrschaftsherren war ihr Herrschaftsgebiet nicht bernisches Untertanenland, begründete das Burgrecht der Herrschaftsherren, das in beiderseitigem Interesse eingegangen war zum Zwecke vereinter Abwehr der Machtanspriuche Kiburgs und Savoyens, keine Hoheitsrechte der Stadt über das Gebiet der Herrschaftsherren, wie es auch diese nicht zu Untertanen der Stadt machte. Die Lage wird dadurch noch komplizierter, daß durch Kauf und auf andere Weise ein Teil der Twingherrschaften auf stadtbernische bürgerliche Geschlechter überging. Stürler stellt im einzelnen die Befugnisse dar, um die sich der Streit drehte (Heerbann, Steuern oder Tellen und Fuhrungen, Abzugsgelder, Wildbann, Fischenzen, Bienenflug u. a. m.), sucht dann die Gerichtsverhältnisse zu entwirren mit Einschluß der Sondergerichte und der für die Steigerung des bernischen Machteinflusses besonders bedeutsamen Schiedsgerichte, wobei auch sehr interessante Angaben über die Straf- und Zivilrechtspflege, über die Anwendung des Gottesurteils (gerichtlicher Zweikampf 1288, heißes Eisen 1315, Bahrprobe 1503, ja noch nach 1648), über einzelne merkwürdige Rechtsstreitigkeiten gemacht werden. Es folgt ein Abschnitt über den bekannten, von dem bernischen Stadtschreiber Thüring Fricker ausführlich berichteten Twingherrenstreit von 1470/1, der mit einer gewissen Einschränkung, nicht aber mit der Beseitigung der Twingherrschaften endete, die vielmehr bis 1798 fortbestanden, ein Kapitel, mit dem der Verfasser die Behandlung der neuerdings viel beachteten Volksanfragen verbindet. Der Periode vom Twingherrenstreit bis zum Untergang des alten Bern ist der folgende, vierte Abschnitt gewidmet. Zunächst lernen wir die vier Venner oder Bannerträger bzw. Steuereinzieher der vierBerner Stadtviertel (wahrscheinlich seit 1295) kennen, seit ca. 1450 aus den Gesellschaften der Gerwer, Schmiede, Pfistern und Metzger genommen und eben dadurch zu besonderer Bedeutung erhoben, daß sie die Militärund zum Teil auch die Gerichtsverwaltung der vier Landgerichte übertragen bekamen. Weiter die Freiweibel, von denen in den einzelnen Landgerichten je nach deren Größe drei, zwei oder einer als Unterbeamte der Venner tätig waren. Durch den Vertrag von 1471 steigerte sich ibre Machtstellung gegenüber den Twingherren ganz bedeutend. Das Recht der Herrschaftsleute gelangt auf Grund der im 16. bis 18. Jahrhundert aufgezeichneten, inhaltlich jedoch z. T. weit älteren Gerichtsordnungen zur Darstellung, die sich aber nur mit Prozeß- und Strafrecht befassen. Das Privatrecht fehlt darin gänzlich; in den Landgerichten kam subsidiär das Berner Stadtrecht zur Anwendung. Wir erhalten einen guten Einblick in die Organisation der Niedergerichte mit dem Twingherrn als Gerichtsherrn, mit dem Ammann als seinem Gerichtshalter und den zwölf Gerichtssäßen oder Geschworenen, die ordentlicherweise als Wochen-, außerordentlicherweise als Gastgericht zusammentraten. Wir lernen aber auch das infolge des Wettbewerbs der Gerichtsbarkeit 
des Großen Rats zur bloßen Form herabsinkende Verfahren an den Landgerichten kennen. Daran reiht sich eine ausführliche Darstellung des bernischen Staatsrechts mit Einschluß des Kirchen- und Gemeinderechts. Alles in allem erhält man aus dieser Darstellung wie aus anderen älteren und neueren Ursprungs durchaus den Eindruck, daß die „demokratische Aristokratie" Berns ein überaus wohlgeordnetes, durch das Vertrauen der breitesten Volksschichten getragenes Gemeinwesen war, das 1798, ähnlich wie in unseren Tagen die Monarchie in Deutschland, mit nichten an innerer Fäulnis zusammengebrochen ist - Tyrannen gab es auch da nicht zu stürzen -, sondern lediglich das Opfer äußeren Drucks und eines Zeitgeistes wurde, der ein fremdes Gewächs war und vornehmlich in der Neuerungssucht gewisser Kreise Nahrung fand, denen eben von Zeit zu Zeit alles Gewordene, mag es seine Daseinsberechtigung noch so sehr erwiesen haben, zum Überdruß wird. Es hat einer Entwicklung von Jahrzehnten bedurft, bis wieder Zustände sich herausbildeten, die praktisch annähernd ebenso zu befriedigen vermochten. Ein umfangreicher Anhang bisher ungedruckter Marchbriefe, Gerichtsweistümer und anderer teils aus dem Berner Staatsarchiv, teils aus dem Herrschaftsbuch von Dießbach und anderswoher stammender Quellen erhöht den Wert der schönen Arbeit, die vielleicht durch strengere Beschränkung auf den eigentlichen Gegenstand und dadurch noch etwas gewonnen hätte, daß bei den einzelnen behandelten Materien der bisherige Stand der Forschung erkennbar gemacht worden wäre.

Die in erster Linie wirtschaftsgeschjchtliche Dissertation von Bein über die Leinwandweberei im Kanton Bern verdient deshalb eine kurze Erwähnung an dieser Stelle, weil sie eine nicht unwichtige rechtsgeschichtliche Nebenfrucht gezeitigt hat. Sie weist nämlich nach, daß in Bern, aber auch in den anderen eidgenössischen Orten, die kein Zunftregiment, vielmehr eine aristokratisch-oligarchischeVerfassung hatten, und die von Zunftzwang und Zentralisation des Handwerks in der Stadt nichts wußten, ländliche, unpolitische, also rein wirtschaftliche Handwerksverbände, von $\mathrm{Be}$ in als Meisterschaftsverbände bezeichnet, bestanden haben. Namentlich im deutschen und vor allem im ackerbautreibenden Teil des ehemaligen Standes Bern kommen sie vor. Sie sind uns vom 16.-19. Jahrhundert bezeugt, waren aber bei der bäuerlichen Landbevölkerung wenig beliebt, da sie diese hinderten, auch für den Markt zu arbeiten. Im Bauernkrieg verlangte deshalb einer der 27 Artikel ihre Aufhebung Doch wurden sie nachher wieder zugelassen, wenn auch die bernische Regierung diese Dinge mehr gehen ließ, als daß sie sich ihrer annahm. Die Arbeit von Bein stützt sich wesentlich auf archivalisches Material. In einem Anhang sind Handwerksbriefe teils abgedruckt, teils nambaft gemacht und zwar mit genauer Angabe der Archivsignatur und nicht bloß für Bern, sondern auch für Luzern, Solothurn und Freiburg i. Ü.

Langenthal unter der Zwingherrschaft des Klosters St. Urban betitelt sich eine rechts- und wirtschaftsgeschichtliche Studie, die Karl Geiser anläßlich des Zusammentritts der Schweizerischen geschicht- 
forschenden Gesellschaft in seinem Heimatorte dessen Geschichte gewidmet hat. Sie beansprucht mehr als lokales Interesse. Denn sie entwirft ein scharfumrissenes Bild der Entwickelung einer bernischen Landgemeinde von den Zeiten der mittelalterlichen Grundherrschaft an bis zum Anbruch der Gegenwart. Selbst da, wo sie nicht eben neue Aufschlüsse gibt, wie etwa in der Darstellung des Gemeinderechts mit seiner zur Realgemeinde sich verengernden Bursame und seinen mehr und mehr auch die bloßen Hintersäßen mitumfassenden Burgern, die in Langenthal schon seit dem 16. Jahrhundert der Gemeinde zugehörten, bei ihr armengenössig waren, aber nach und nach sogar Nutzungsrechte an der Allmend erhielten, vertieft sie und präzisiert sie unsere Kenntnis. Vor allem aber vermittelt sie eine anschauliche Vorstellung davon, wie die Grundherrschaft des seit 1420 unter luzernischer Oberhoheit stehenden und deshalb bis 1848 erhaltengebliebenen Klosters St. Urban seit dem Übergang der Landgrafschaft Burgunden an die Stadt Bern im Jahre $1406 \mathrm{zu}$ einer Twingherrschaft im spezifisch bernischen Sinne wurde, bei der der Twingherr Twing und Bann, die niederen Gerichte, den Kirchenpatronat $u$. a. behielt, aber nur im alten Umfange, ohne Ausdehnungsmöglichkeit, indes Bern außer seiner Hochgerichtsbarkeit und seiner mehr und mehr ausgebauten Landeshoheit ein Bußenstrafrecht entwickelte zur Ahndung der Übertretung seiner zahlreichen Religionsund Sittenmandate. Dafür, wie allmählich die Niedergerichtsbarkeit der Twingherren abstarb, wie sich die Herrschaft des Klosters immer deutlicher auf das Hofrecht beschränkte, wie schließlich zur Zeit der Helvetik die Twingherrlichkeit einseitig, während der Restaurationsperiode im Jahre 1819 in freundlichem gegenseitigen Einverständnis beseitigt wurde, indes die Rechte gegenüber den Einzelnen, wie Bodenzinsen, Ehrschätze, Zehnten, sich noch bis zur obligatorischen Ablösung von 1845 behaupteten, ist Geisers Abhandlung überaus lehrreich. Eine Anzahl noch ungedruckter Dokumente sind ihr beigegeben, besonders der Twingrodel von 1530 und dessen beachtenswerte Revision von 1669 .

Aber auch noch einen anderen rechtsgeschichtlich nicht uninteressanten Beitrag enthält die Festschrift der Langenthaler Tagung, nämlich F. Bühlmanns Aufsatz über das Landgericht Konolfingen zur Zeit des Überganges und der helvetischen Republik. Er ist ein kleiner, aber sehr vielsagender Ausschnitt aus der Geschichte des Untergangs des alten Bern und der alten Eidgenossenschaft überhaupt, der hier geboten wird. Rechtshistorisch in Betracht kommt dabei namentlich der Abschnitt über den Streit betreffend die Primizien, die dem Pfarrer zu entrichtenden Erstlingsgaben. Materiell waren sie nicht eben von Bedeutung, wurden sie doch für Konolfingen auf bloß 25 Mütt im Wert von $200 \mathrm{~L}$. veranschlagt. Das Landvolk betrachtete sie als durch die helvetische Gesetzgebung beseitigt, trotzdem ein Gesetz von 1799 ihre Weiterentrichtung geboten hatte. Es leistete Widerstand und suchte es um des Grundsatzes willen zur Beseitigung der verhaßten, übrigens nicht sowohl den Boden als Grundlast als vielmehr die Familien treffenden Abgabe zu bringen. Doch die Regierung gab nicht nach. Es kam zu 
einer militürischen Besetzung, die überaus verbitternd wirkte und den Betroffenen an die $10000 \mathrm{~L}$. Kosten verursachte. Die Primizien blieben in Geltung und sind erst durch das Gesetz vom 20. Dezember 1845 betreffend die Aufhebung der Feudallasten beseitigt worden und zwar unentgeltlich, während für andere Lasten eine mäßige Ablösungsentschädigung festgesetzt wurde. Ich muß es mir versagen, auf den übrigen Inhalt der Bühlmannschen Abhandlung einzutreten, obschon er wie alles, was jene Zeit des Zusammenbruchs und der Revolution betrifft, heutzutage der Beachtung besonders wert erscheint.

Gerade deshalb begrüßen wir es nicht bloß im Interesse der Schweizerischen Ruechtsgeschichte, sondern auch in dem der Deutschen und der Geschichte des Staatsrechts überhaupt freudig, daß nunmehr auch das Staatsrecht der Helvetik und der Mediation eine, sagen wir es gleich, ganz ausgezeichnete Bearbeitung gefunden hat. Das Werk des Basler Privatdozenten Eduard His, eines Ururenkels des Basler Oberstzunftmeisters Peter Ochs, der im Jahre 1797/8 im Einverständnis mit Reubell und dem General Bonaparte die Revolutionierung der Schweiz in Gang brachte und, zumal in der ersten Zeit, als Präsident der Basler kantonalen Nationalversammlung und des ersten Senats der helvetischen Republik zu den markantesten Persönlichkeiten und hervorragendsten Staatsmännern der neuen Ära gehörte, bewältigt den fast überreichen Stoff zum ersten Male rechtsgeschichtlich und mit dem Anspruch auf eine gewisse Vollständigkeit. Ausgebreitetes Studium der von Johannes Strickler vortrefflich herausgegebenen Akten, aber auch noch ungedruckten Materials, eine jeden einzelnen Punkt bis in seine letzten Beziehungen aufhellende, doch niemals aufdringliche Gelehrsamkeit, erfreuliche Gestaltungskraft und ein gefälliger, überaus lesbarer Stil heben das Buch, an dem auch die Reife und Ruhe des Urteils sowie die vornehm-wissenschaftliche, streng objektive Art der Behandlung stark umstrittener Fragen wohltuend auffällt, weit über das Durchschnittsmaß empor und gestalten seine Lektüre recht eigentlich zum Genuß. Der Reichtum seines Inhalts bringt es freilich mit sich, daB man ihm selbst in einer ausführlichen Besprechung, geschweige denn im Rahmen einer bloßen Sammelanzeige wie der gegenwärtigen unmöglich gerecht werden kann. Ich muß mich auf das Notwendigste beschränken. Als erstes Kapitel wird eine kurze Übersicht über den Gang der Dinge von 1798 bis 1813 vorausgeschickt, also eine Verfassungsgeschichte der Helvetik, die 1798 als Einheitsstaat begann und, seit 1802 Bundesstaat, 1803 endete, um durch den Staatenbund von Napoleons Gnaden vermöge dessen Vermittlungsakte abgelöst zu werden, bis auch dieser 1813 infolge der Siege der Verbündeten ein Ende nahm. Weiter folgt ein zweites Kapitel, der naturrechtlichen Theorie gewidmet, mit deren Gedanken vor allem die jede Anknüpfung an das geschichtlich Gewordene verschmähende Helvetik arbeitete, also namentlich den Menschen- und Bürgerrechten mit ihrer engli sch-nordamerikanischen Wurzel und ihrer französischen Ausgestaltung. Und nun wird in weiteren 20 Kapiteln die Rüstkammer des Staatsrechts der beiden Perioden auf ihren ganzen 
Inhalt hin systematisch durchforscht, also namentlich von Staatsform, Staatsangehörigkeit und Staatsgebiet, von Volkssouveränität, verfassunggebender Gewalt, Trennung der Gewalten und deren Organisation sowie von der Volksvertretung (Repräsentativprinzip) gehandelt, aber auch von Rechtsgleichheit, Religionsfreiheit, Preßfreiheit, vom freien Versammlungs- und Vereins- sowie vom Petitionsrecht, von der Niederlassungs-, Handels- und Gewerbefreiheit, von der Freiheit des Eigentums und der Lastenfreiheit des Grundes und Bodens, endlich von der Steuer-, Wehr- und Schulpflicht. Überall wird angeknüpft an die Zustände der alten Eidgenossenschaft, danin der Bestand der importierten Ideen festgestellt, besonders wie er sich in Frankreich herausgebildet hatte, hierauf der Brechungen gedacht, die sich das fremde Gewächs bei seiner Verpflanzung auf schweizerischen Boden doch hie und da gefallen lassen mußte, weiter die gesetzgeberische Durchführung und praktische Verwirklichung im helvetischen Einheits- bzw. Bundesstaat dargestellt und endlich nachgewiesen, wie unter der Herrschaft der Mediationsakte das meiste auf die Gleise der kantonalen Entwicklung hinübergeschoben wird. Die sklavische politische und gedankliche Abhängigkeit von Frankreich, die wilde Gährung, in der sich damals auch die schweizerischen Verhältnisse befanden, aber auch die allmähliche Konsolidierung und die weitreichende, bis auf den heutigen Tag grundlegende Bedeutung der immerhin von anerkannt hervorragenden Köpfen ins Werk gesetzten völligen Umgestaltung des schweizerischen Gemeinwesens kommt dem Leser noch ganz anders als bisher, insonderheit mit einer bis in die Einzelheiten vordringenden Klarheit und Anschaulichkeit zum Bewußtsein. Von Gegenständen, die, ohne daß es aus den oben mitgeteilten Kapitelüberschriften ersichtlich wäre, von His mitbehandelt werden, erwähne ich namentlich das Gemeindewesen mit seinem Dualismus von Gemeindegutsgenossenschaft (Bürgerschaft) und von Aktivbürgergemeinde (Einwohnergemeinde), einer Lösung, die ausnahmsweise AltEidgenössisches mit Modern-Französischem zu verbinden verstand, ferner das Gemeindebürgerrecht sowie der strenggenommen über die eigentliche Aufgabe hinausgehende, aber höchst interessante und den Verfasser auch auf dem Gebiete des deutschen bzw. schweizerischen Privatrechts wohl bewandert erweisende Abschnitt über die Grund. entlastung. Hatte der Verfasser bezüglich des Steuerrechts in Georg Schanz, bezüglich der Religionsfreiheit in Eduard Herzog treffliche Vorgänger, so stellt gerade die Art und Weise, wie er in der Herausarbeitung der leitenden Ideen über jenen, in der Gesamterfassung des Verhältnisses von Staat und Kirche über diesen herausgekommen ist, den Wert seiner Leistung in helles Licht. Der Absohnitt über die Religionsfreiheit gehört überhaupt zu den schönsten und inhaltreichsten des Buchs. Hoffen wir, daß dieses trotz der Ungunst der Zeiten auch außerhalb der Schweiz die ihm gebührende Beachtung finden werde, und daß es dem Verfasser vergönnt sei, in einem zweiten Bande in nicht allzuferner Zeit das Staatsrecht der Restauration (bis 1830) und das der Regeneration (bis 1848) darzustellen. Daß er feißig am Werke ist, 
lehrt sein seither erschienener Beitrag zu der Festgabe für den Basler Juristentag von 1920: Amerikanische Einflüsse im Schweizerischen Verfassungsrecht. Er beschäftigt sich mit der in den vierziger Jahren des vergangenen Jahrhunderts angebahnten und 1848 ausgeführten Übernahme der amerikanischen Bundesstaatsform und des amerikanischen Zweikammersystems in die Schweizerische Bundesverfassung.

Jedoch noch ein größeres Ereignis ist in diesem Jahre auf dem Gebiete der Schweizer Rechtsgeschichte zu verzeichnen: das Erscheinen einer Schweizerischen Verfassungsgeschichte von Andreas Heusler. Über schweizerische Rechtsgeschichte wird seit Jahrzehnten an den schweizerischen Universitäten gelesen. Staats- und Rechtsgeschichten einzelner Kantone, insbesondere Zürichs, Luzerns, der innerschwẹizerischen Länder, aber auch Darstellungen der Geschichte des schweizerischen Bundesrechts liegen seit langem vor; man braucht nur an die Namen Bluntschli, Blumer, v. Segesser, Johannes Meyer zu erinnern. Die Geschichte der Rechtsquellen und des Privatrechts hat Eugen Huber behandelt. Aber eine Gesamtrechtsgeschichte der Schweiz steht noch aus; nicht einmal eine die Entwicklung des Bundesrechts und der Verfassurfg der einzelnen Orte bzw. Stände zusammenfassende Verfassungsgeschichte der Eidgenossenschaft gab es bis jetzt. Nachdem er mit der Verfassungsgeschichte seiner Vaterstadt Basel begonnen, im Laufe seines an Arbeit und Erfolgen ungewöhnlich reichen Lebens eine Geschichte des deutschen Privatrechts und einen Grundriß der deutschen Verfassungsgeschichte geschrieben und erst neulich eine kurzgefaßte Geschichte Basels ${ }^{1}$ ) veröffentlicht hat, krönt der Basler Altmeister nunmehr sein Lebenswerk noch mit der ersten Schweizerischen Verfassungsgeschichte, genauer mit einer für weitere Kreise historisch und juristisch, vor allem aber auch vaterländisch Interessierter bestimmten Einführung in sie. Das Buch gibt in sorgfältig revidierter und wohl auch da und dort erweiterter Gestalt Vorlesungen wieder, die der Verfasser in seinem 82. Lebensjahre während zweier Semester an der Universität Basel gehalten und auf vielfachen Wunsch nunmehr als Sechsundachtzigjähriger noch herausgebracht hat. Schon deswegen eine in der Geschichte der Wissenschaft fast einzig dastehende Leistung, die allen denen zu denken geben muß, die es nicht wahr haben wollen, daß die geistige Schöpferkraft nicht vor schablonenhaft aufgestellten Durchschnittsaltersgrenzen haltmacht, daß vielmehr das Genie aller Normalerfahrungen und -vorschriften spottet! Gewiß würde ein Jüngerer, würde Heusler selbst in früheren Jahren die Aufgabe in der einen oder anderen Hinsicht anders angefaßt, würde er seinen Bau vielleicht unmittelbar aus den Quellen oder vielmehr aus einer mit Vorbedacht getroffenen Auswahl von solchen vor den Augen seiner Leser haben entstehen lassen, würde er sie mehr zur Mitarbeit und zu eigenem Urteil gebracht haben, während es ihm nunmehr vor allem darauf ankommt, die Summe seines Wissens und seiner Anschauung von diesem Gegenstand, der ihn sein ganzes Leben

1) Siehe diese Zeitschr. XL, 1919, Germ. Abt. S. 372, $386 \mathrm{ff}$. 
hindurch beschäftigt hat, der Mit- und Nachwelt zu erhalten. Aber gerade daß er sich etwas freier gehen läßt und mit seinem persönlichen Urteil nicht zurückhält, das übrigens immer geistvoll und stets von einem ehrlichen Streben nach strengster Objektivität getragen, wenn auch höchst subjektiv geprägt ist, macht einen Hauptreiz des Buches aus. Komposition und Ökonomie aber verdienen rückhaltlos Bewunderung. Der Schwierigkeit, die Verfassungsgesehichte eines Ganzen darzustellen, dessen Teile älter als dieses sind, wird Heusler spielend dadurch Herr, daß er wenigstens vorläufig und in Kürze die voreidgenössische Verfassungsentwicklung jedes einzelnen Orts bei dessen Hinzukommen gibt, um dann am Schlusse der beiden Teile die Verfassung der regierenden Orte, der Zugewandten, der Untertanenlande und gemeinen Vogteien im Zusammenhang ausführlicher nachzuholen. Auch hat er den Stoff mit wahrhaft erstaunlicher Gleichmäßigkeit bewältigt. Am Anfang fünfzig Seiten über die Zeit von der alamannischen und burgundischen Besitznahme an bis zu der Entstehungsgeschichte des Bundes der drei Waldstätte, am Schluß hundert Seiten über die Entwicklung des 19. Jahrhunderts von der Helvetik bis zur Bundesverfassung von 1848; dazwischen in zwei annähernd gleichen Teilen Bundes- und Ortsrechtsgeschichte bis zum Abschluß der dreizehnörtigen Eidgenossenschaft und die Zeit der alternden Eidgenossenschaft von der Glaubenstrennung bis zum Untergang im Jahre 1798. Dafür, daß er letztere nicht hat zu kurz kommen lassen, muß man dem Verfasser besonders Dank wissen. Es ist, gleichwie der entsprechende Teil der deutschen Rechtsgeschichte, ein alles andere als erhebender Abschnitt. Heusler gesteht auch, daß ihm, weil er nicht als "landfremder Rechtshistoriker sine ira et studio", sondern mit der inneren Anteilnahme des Patrioten schrieb, die Ausarbeitung gerade dieses Stücks besonders sauer geworden sei; wer jetzt angesichts des Zusammenbruchs Deutschlands dieselbe deutsche Rechtsgeschichte, die noch vor kurzem in eine glanzvolle Gegenwart als Krone des Ganzen ausmündete, vorzutragen hat, besitzt Verständnis für die Hemmungen, die innere, persönliche Anteilnahme auch an einem historischen Stoff seiner wissenschaftlichen Bewältigung bereiten kann. Immerhin haben diese drei Jahrhunderte schweizerischer Verfassungsgeschichte für den schweizerischen Bearbeiter das für sich, daß das spezifisch Schweizerische in ihnen mehr und mehr hervortritt, während die Verfassungsgeschichte bis zum Schwabenkrieg eben im wesentlichen noch als deutsche Verfassungsgeschichte, wenn auch überwiegend als deutsche Teilverfassungsgeschichte sich darstellt. Eben deshalb hat man sich in der Schweiz mit diesen drei Jahrhunderten der alternden Eidgenossenschaft von jeher ganz anders beschäftigt und für sie ganz anders vorgearbeitet als in Deutschland für die entsprechenden Jahrhunderte der deutschen, was Heusler die Arbeit ungemein erleichtern mußte. Überhaupt geht ja das Buch nicht auf Neuerforschung, sondern auf Zusammenfassung, auf Vermittlung eines Gesamtüberblicks aus, was nicht ausschließt, daß es in Einzelheiten Neues bietet, Lücken ausfüllt, und daß der Verfasser das Ganze mit der Selbständigkeit erfaßt und geformt hat, die dem Meister 
auch da eigen ist, wo er mit Bausteinen arbeiten muß, die Andere geformt haben. Das Entscheidende ist, daß hier der Stoff in seinem ganzen Umfange zum ersten Male durchaus juristisch, rechtshistorisch erfaßt ist. Man kann im Zweifel darüber sein, ob die Grenzen zur politischen Ge. schichte hin überall richtig gezogen sind. Es findet sich manches in dem Buche, was strenggenommen nicht oder nicht in dieser Ausführlichkeit in eine Verfassungsgeschichte hineingehört. Stellenweise nimmt es sich aus wie eine Schweizergeschichte unter besonderer Berücksichtigung der Geschichte der Institutionen. Aber seinen Zweck, auf weitere Kreise zu wirken, wird es gerade deshalb um so besser erfüllen. Auf der anderen Seite hat Heusler freilich in der Beschränkung auf seinen Gegenstand des Guten fast wieder zu viel getan. Mit den Quellen und ihrer Geschichte befaßt er sich gar nicht, kaum daß er hie und da einmal auf eine entlegenere Quelle hinweist und andeutet, wo der Beleg für das Gesagte zu finden ist. Nicht nur dem deutschen Leser, sondern vor allem auch dem schweizerischen Anfänger wird das große Schwierigkeiten bereiten. Zum mindesten hätte in einer Anmerkung darauf hingewiesen werden müssen, daß der Leser die Quellengeschichte bei Huber findet. Auf Einzelheiten einzugehen, verbietet mir der Mangel an Raum; noch weniger als dem Hisschen Buche vermag diese Sammelanzeige der Bedeutung des Heuslerschen gerecht zu werden. Auch da, wo man nicht mitgehen kann, fesselt Heusler durch die Lebendigkeit seines Anschauungsvermögens und durch seine Darstellung sowie durch sein temperamentvolles Urteil. Erfrischend wirkt die Deutlichkeit, mit der er sein politisches Bekenntnis und sein Baslertum durchblicken läßt, die militärische und politische Größe Berns bewundert und den Zürchern am Zeuge flickt, wo er kann, selbst wenn er sie etwas spitz gate Juristen nennt, die sich auf ihren Vorteil versteben. Auch darin tritt nur zutage, daß er nicht als ,landfremder Rechtshistoriker" die Feder geführt hat, sondern als echter. Kidgenosse vom alten, guten Schlag.

Am Anfang dieses Jahres sprach ich am Schlusse einer in der Berliner Akademie bei festlichem Anlaß gehaltenen Rede die Hoffinung aus, daß eine Schweizerische Rechtsgeschichte, die den Beziehungen zur Deutschen nicht minder gerecht werde als die bisherige rechtsgeschichtliche Forschung der Schweiz, uns bald möge beschert werden. Wenigstens für die Verfassungsgeschichte ist dieser Wunsch über Erwarten rasch mit Heuslers Schweizerischer Verfassungsgeschichte in Erfüllung gegangen. Wie es sich bei diesem Verfasser von selbst versteht, und wie gleich die erste Seite mit dem Satze ,Aus germanischen Grundlagen hat sich das schweizerische Recht entwickelt" es deutlich zum Ausdruck bringt, bewegt sich Heuslers Darstellung durchaus in der Richtung, in der ich eine eigene Sohweizerische Rechtsgeschichte als berechtigt, ja als dringend erwünscht bezeichnet hatte. Ohne im übrigen meine gewissermaßen eine Einleitung darstellende Leistung irgendwie in Vergleich mit Heuslers Tat rücken zu wollen, darf ich vielleicht doch am Schlusse dieser Anzeige den Leser, der sie noch nicht zu Gesicht bekommen hat, auf sie hinweisen. Als Germanist, der von seiner heimatlichen, eben der Schweize- 
rischen Rechtsgeschichte ausgegangen ist, erstatte ich seit 25 Jahren an dieser Stelle Bericht über Literatur zur Schweizerischen Rechtsgeschichte. Als in der Schweizerischen, meiner heimatlichen Rechtsgeschichte noch heute wurzelnder Germanist glaubte ich vor einer gelehrten Körperschaft, der seit den Tagen Friedrichs des Großen immer Schweizer angehört haben, und die diese Beziehungen stets besonders pflegte, reden zu sollen über die Schweiz in der Deutschen Rechtsgeschichte, nicht bloß um einmal einen Anfang zu machen mit dem Nachweis der Rolle, die einzelne Landschaften in der deutschen Gesamtrechtsgeschichte gespielt haben, sondern namentlich auch um zu zeigen, in welch weitgehendem Maße die Deutsche Rechtsgeschichte mit schweizerischem Stoff arbeitet, und wieviel sie der Mitarbeit schweizerischer Forscher verdankt. Gerne bekenne ich, daß dabei auch persönliche Gründe mitspielten. Je älter man wird, um so mehr fühlt man sich gedrängt, zu seiner ersten Arbeitsliebe zurückzukehren. Diese aber war und bleibt für mich die Zürcherische, die Schweizerische Rechtsgeschichte. Vollends inmitten der wilden, sinnlosen Zerstörung, die mich umgibt, und in einer Welt, die überheblich meint, den Zusammenhang mit ihrer Vergangenheit entbehren zu können, richtet sich der Blick bei der Arbeit unwillkürlich nach dem engeren Vaterlande, das zwar einst vorübergehend auch solchem Wahne erlegen ist, aber sich inzwischen längst von ihm erholt hat, um ihm hoffentlich nie wieder zu verfallen. Sanctus amor patriae dat animum.

UIrich Stutz.

\section{Josef Lappe, Freiherr vom Stein als Gutsherr auf Kappen-} berg. Münster i. W., Aschendorff 1920. IX und 218 S. $8^{\circ}$.

Der bekannte Erforscher westfälischer Rechts- und Agrargeschichte fand bei seiner Arbeit „Entstehung und Feldmarkverfassung der Stadt Werne" (Zeitschr. f. Gesch. u. Altertumsk. Westfalens 67 [1918]) im Archiv der Prämonstratenserpropstei Kappenberg auch den vollständigen Briefwechsel, den ihr erster Privateigentümer nach der Säkularisation, der Freiherr Karl vom Stein, von ihrem Erwerb bis zu seinem Tode (1816-31) mit seinen dortigen Verwaltern („Rentmeistern“), Leutnant Geisberg und Oberförster Poock, geführt hat. Diese Quelle und verwandte Akten, wie namentlich die ausführlich belegten Wirtschafts. rechnungen, sind in dem vorliegenden Buch in einer Weise verwertet, die das wenige bisher besonders durch Pertz und Lehmann über Stein als Privatwirtschafter Bekannte glücklich ergänzt. Der Jurist und der Nationalökonom werden vielleicht bisweilen den engen Anschluß des Verfassers an sein Material bedauern, der nicht nur Wiederholungen bedingt und vor Belanglosigkeiten (wie der Journalnotiz vom 6. Februar 1830 S. 137) nicht zurückscheut, sondern auch die Verhältnisse nicht immer in der wünschenswerten technischen Klarheit erscheinen läßt.

Gleich der erste Abschnitt über die Entstehung der Standesherrschaft Kappenberg-Scheda, mit der Stein 1825 durch königliche Ver- 